\title{
The Significance of Mulches on Growth, Yield and Quality of Summer Sesamum (Sesamum indicum L.) in Relation to Weather Parameters
}

\author{
S. G. Mundhe*, D. A. Sonawane and P. B. Suryavanshi
}

Department of Agricultural Meteorology, College of Agriculture, Pune-411 005, Mahatma Phule Krishi Vidypeeth Rahuri 413 722, Maharashtra, India

*Corresponding author

\section{A B S T R A C T}

\section{Keywords}

Mulch, Weather,

Yield, Correlation,

Sesamum

Article Info

Accepted:

04 September 2019

Available Online:

10 October 2019
The field experiment was conducted during summer 2014 on sesamum, Sesamum indicum L. with an objective to study the mulches on growth, yield and quality of summer sesamum in relation to weather parameters. The results of the study indicated that all the treatments were significantly influenced. Amongst the treatments, sowing of sesamum on the application of sugarcane trash mulch @ $5 \mathrm{t} \mathrm{ha}^{-1}$ was found significantly superior in plant height $(101.47 \mathrm{~cm})$, number of functional leaves plant ${ }^{-1}(92.68)$, leaf area plant ${ }^{-1}\left(8.83 \mathrm{dm}^{2}\right)$, plant spread plant ${ }^{-1}(43.19 \mathrm{~cm})$, number of branches plant $^{-1}(6.78)$, number of capsules plant ${ }^{-1}$ (58.81), dry matter plant ${ }^{-1}(20.35$ g), seed weight plant $^{-1}(4.02 \mathrm{~g})$ and grain yield (886.76 kg ha $\mathrm{kg}^{-1}$, gross monetary returns (70939 Rs.ha ${ }^{-1}$ ), net monetary returns (33779 Rs.ha ${ }^{-1}$ ). The study of Correlation coefficient between weather parameters and plant growth contributing characters of summer sesamum are positively significant in all mulches.

\section{Introduction}

Sesamum is an east Indian important oilseed crop belongs to family Pedaliaceae and the genus Sesamum. Even though it is grown all over the world for its importance in food, medicine and industries. The crop is highly drought tolerant, grows well in most kind of soils, regions and is well suited to different crop rotations. In reality, sesame is mostly grown under moisture stress with low management input by small holders. Among the oilseed crops, sesame ranks first for having the highest oil content generally varies from 48 to 52 per cent and contains $6355 \mathrm{kcal} \mathrm{kg}^{-1}$ energy in seeds (Kumar and Goel, 1994).

The seed is also rich source of proteins (20$28 \%$ ), sugars $(14-16 \%)$ and minerals $(5-7 \%)$ particularly nutrients like calcium $(1.31 \%)$ and phosphorus (Dinosa and Gupta, 1993). Hence, sesamum is known as "Queen of oilseed 
crops" by virtue of the excellent quality of oil as used for domestic purpose. It is one of the most important ancient edible crops grown in India next to groundnut and rapeseed-mustard. India is now considered as the basic centre of origin (Rathore, 2005).

The highest production is in China with higher productivity. India ranks first in area (45\%), production $(32 \%)$ and export $(40 \%)$ of sesamum in the world. The area under sesamum in India was 18.1 lakh ha with the production of 7.32 lakh tonnes having productivity of $354 \mathrm{~kg} \mathrm{ha}^{-1}$ during 201213(Anonymous, 2012).

Study was designed to see the significance mulches on growth, yield and quality of summer sesamum (Sesamum indicum L.) in relation to weather parameters.

\section{Material and Methods}

The field experiment was conducted during summer season of 2014 on sesamum (cv.) 'JLT-408' at Experimental Farm of Agril. Meteorology Department, College of Agriculture, Pune, Maharashtra State.

Climatologically the area is under subtropical, semi-arid region and agro climatologically is under plain zone of Maharashtra.

The average annual rainfall is about $714 \mathrm{~mm}$, which gets received mostly during months from June to September (From south-west monsoon). The remaining rainfall receives during period from October to December.

The experiment was laid out in split plot design with three replications. Twelve treatment combinations were formed considering main plot treatments comprise four planting layouts and three sub-plot treatments of mulches. The details are listed below.

\section{Main plot treatments (layouts)}

$\mathrm{P}_{1}$ :Ridges and furrow at $45 \times 10 \mathrm{~cm}^{2}$

$\mathrm{P}_{2}$ :Ridges and furrow at $30 \times 15 \mathrm{~cm}^{2}$

$\mathrm{P}_{3}:$ Flat bed at $45 \times 10 \mathrm{~cm}^{2}$

$\mathrm{P}_{4}:$ Flat bed at $30 \times 15 \mathrm{~cm}^{2}$

\section{Sub plot treatments (mulches)}

$\mathrm{M}_{1}:$ No mulch

$\mathrm{M}_{2}$ :Soybean straw mulch @ $5 \mathrm{tha}^{-1}$

$\mathrm{M}_{3}:$ Sugarcane trash mulch @ $5 \mathrm{tha}^{-1}$

Thinning and gap filling was done on 14 DAS to achieve the required plant population. The observations were recorded 14 days interval from 28 DAS to 84 DAS and at harvest. Five sample plants of sesamum from each treatment plot were selected at random and labeled for biometric observations. The observations like plant height $(\mathrm{cm})$, number of functional leaves plant ${ }^{-1}$, leaf area $\left(\mathrm{dm}^{2}\right)$ plant ${ }^{-}$ 1 , plant spread $(\mathrm{cm})$ plant-1, number of branches plant ${ }^{-1}$, number of capsules plant ${ }^{-1}$ and dry matter $(\mathrm{g})$ plant $^{-1}$ were recorded. The crop was harvested separately as per the treatment when the leaves and stem were turned yellow, processed and the seed yield was recorded.

The weather elements play an important role in success or failure of the crop. The weather requirement varies differently in the different phenophase and hence the study of individual weather element prevailed during different phenopheses was conducted by studying the degree of association between seed yield and total biomass versus weather elements. The weather elements considered canopy temperature $\left({ }^{0} \mathrm{C}\right)$, growing degree days, photosynthetically active radiation and albedo 
(\%) were included in this study. The correlation coefficients for each treatment were estimated through Pearsmans correlation techniques.

Data were analyzed using standard analysis of variance (ANOVA) technique and means were separated using least significant difference comparisons.

\section{Results and Discusion}

\section{Growth characters}

\section{Plant height (cm)}

Among the mulch treatments, the maximum plant height $(101.47 \mathrm{~cm})$ was recorded with application of sugarcane trash mulch @ 5 $\mathrm{t} \mathrm{ha}^{-1}$ (Table 1). This may be due to better conservation and efficient use of moisture for plant under sugarcane trash mulch than soybean straw and no mulch. There results are in confirmative with Pawar et al., (2008) and Sonawane (2007).

\section{Number of functional leaves plant ${ }^{-1}$}

The mean numbers of functional leaves plant ${ }^{-1}$ were significantly affected by different mulches. The highest numbers of functional leaves plant $^{-1}$ were observed in sugarcane trash mulch (92.68) as compared to no mulch and soybean straw mulch (Table 1). Similar results were reported by Pawar et al., (2008) and Sonawane (2007).

\section{Leaf area palnt ${ }^{-1}\left(\mathrm{dm}^{2}\right)$}

Application of different mulches showed significant effect on leaf area plant $^{-1}$. The maximum leaf area $\left(8.83 \mathrm{dm}^{2}\right)$ plant $^{-1}$ was recorded with application of sugarcane trash mulch@ $5 \mathrm{t} \mathrm{ha}^{-1}$ as compared to no mulch and soybean straw mulch (Table 1).

\section{Number of branches plant ${ }^{-1}$}

Numbers of branches plant $^{-1}$ were significantly influenced by different planting layouts at all the growth stages. The maximum number of branches plant ${ }^{-1}$ (6.75) which was found significantly. The number of branches plant $^{-1}$ were significantly affected by different mulching.

The maximum number of branches plant ${ }^{1}$ were observed in of sugarcane trash mulch @ $5 \mathrm{t} \mathrm{ha}^{-1}$ (6.78), which is due to better conservation and efficient use of moisture in plant for physiological processes while increasing the simpodial branches of plant and it was found significantly superior to rest of the mulch treatments (Table 1).

Similar findings were reported by Pawar et al., (2008), Sonawane (2007) and Yadav and Patil (2009).

\section{plant spread plant ${ }^{-1}(\mathrm{~cm})$}

There were significant differences in plant spread due to different mulches from 28 days onwards up to harvest (Table 1).

Among the different mulches, the maximum plant spread $(44.35 \mathrm{~cm})$ at harvest was recorded at harvest with the application of sugarcane trash mulch@ $5 \mathrm{t} \mathrm{ha}^{-1}$ and it was found significantly superior to rest of the mulch treatments.

\section{Dry matter plant ${ }^{-1}(g)$}

The mean dry matter accumulation plant $^{-1}$ of sesamum was differed significantly due to different mulches (Table 1). The dry matter accumulation in the treatment of sugarcane trash mulch@5 tha ${ }^{-1}$ was found significantly more at all the crop stages up to at harvest (20.35 g) but it was at par with 28 DAS 


\section{Number of capsules plant ${ }^{-1}$}

Application of different mulches showed significant effect on number of capsules plant ${ }^{-}$

1 . The maximum number of capsules plant ${ }^{-}$ ${ }^{1}$ (58.81) were obtained with application of sugarcane trash mulch@5 t ha ${ }^{-1}$ and was found significantly superior to rest of the mulch treatments during all growth stages (Table 1).

This might be due to better conservation and efficient use of moisture in plant for physiological processes while increasing the number of capsules of sesamum. Similar findings were also noted in Anonymous (1997).

\section{Yield contributing characters}

\section{Seed weight plant $^{-1}(\mathrm{~g})$}

The seed yield plant $^{-1}$ was significantly affected by mulches. The maximum seed yield plant $^{-1}(4.02 \mathrm{~g})$ was obtained with application of sugarcane trash mulch @ $5 \mathrm{t} \mathrm{ha}^{-1}$, which was significantly superior to rest of the mulch treatments (Table 1). Due to better conservation and efficient use of moisture in plant for physiological process while increasing the number of capsules, number of branches plant ${ }^{-1}$ with sugarcane trash mulch might be mulching the resulted in increased seed yield plant $^{-1}$.Similar findings were also reported by Pawar et al., (2008).

\section{Weight of straw plant ${ }^{-1}$ (g)}

The mean straw yield plant ${ }^{-1}$ of sesamum was significantly affected by mulches. The application of sugarcane trash mulch @ $5 \mathrm{tha}^{-}$ ${ }^{1}$ showed favorably higher straw yield plant ${ }^{-1}$. Significantly more straw yield plant $^{-1}$ was obtained with application of sugarcane trash mulch @ $5 \mathrm{t} \mathrm{ha}^{-1}$ (12.84 g) as compared to no mulch and soybean straw (Table 2).

\section{Test weight (g)}

The mean straw yield plant ${ }^{-1}$ of sesamum was significantly affected by mulches. The application of sugarcane trash mulch @ $5 \mathrm{t}$ $\mathrm{ha}^{-1}$ showed favorably higher straw yield plant $^{-1}$. Significantly more straw yield plant ${ }^{-1}$ was obtained with application of sugarcane trash mulch @ $5 \mathrm{t} \mathrm{ha}^{-1}(12.84 \mathrm{~g})$ as compared to no mulch and soybean straw (Table 2).

\section{Yield}

\section{Grain yield $\left(\mathrm{kg} \mathrm{ha}^{-1}\right)$}

The seed yield of sesamum was significantly affected by different mulches. The highest seed yield $\left(886.76 \mathrm{Kg} \mathrm{ha}^{-1}\right)$ was obtained in sugarcane trash mulch@5 t ha ${ }^{-1}$ which was found significantly superior to than other mulch treatments (Table 2).

This might be due to better conservation and efficient use of moisture in plant, while recorded optimum temperature prevailed during flowering resulted in low flower drop and higher fruit setting and is free from pest and disease attack during summer season.

Similar findings were also reported by Anisuzzaman et al., (2009), Pawar et al., (2008), Katmale (2000) and Ghosh et al., (1997).

\section{Straw yield $\left(\mathrm{kg} \mathrm{ha}^{-1}\right)$}

The straw yield was significantly affected by different mulches. The highest straw yield $\left(1574.57 \mathrm{Kg} \mathrm{ha}^{-1}\right)$, (Table 2) was obtained in with sugarcane trash mulch @ $5 \mathrm{t} \mathrm{ha}^{-1}$. It was found significantly superior to rest of the mulch treatments.

\section{Harvest index (\%)}

The harvest index of summer sesamum was found differed significantly due to different 
mulches (Table 2). The maximum harvest application of sugarcane trash mulch @ 5 index $(36.00 \%)$ was noticed with the $\mathrm{t} \mathrm{ha}^{-1}$ than other mulches

Table.1 Effect of mulches on growth characters of summer sesamum

\begin{tabular}{|c|c|c|c|c|c|c|c|c|c|c|c|c|}
\hline \multirow[b]{2}{*}{ Treatments } & \multicolumn{6}{|c|}{ Plant height (cm) } & \multicolumn{6}{|c|}{ Number of functional leaves plant ${ }^{-1}$} \\
\hline & \multicolumn{5}{|c|}{ DAS } & $\begin{array}{l}\text { At } \\
\text { hst }\end{array}$ & \multicolumn{5}{|c|}{ 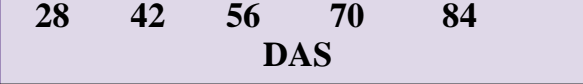 } & $\begin{array}{l}\text { At } \\
\text { Hst }\end{array}$ \\
\hline$M_{1}$ : No mulch & 18.70 & 44.11 & 69.60 & 88.05 & 96.44 & 96.51 & 8.15 & 24.55 & 43.19 & 80.10 & 88.79 & 47.82 \\
\hline$M_{2}$ : Soybean straw & 18.54 & 45.20 & 81.42 & 90.16 & 98.93 & 98.99 & 8.39 & 26.28 & 44.12 & 81.22 & 89.87 & 48.51 \\
\hline$M_{3}$ : Sugarcane trash & 18.73 & 46.56 & 85.78 & 94.93 & 101.09 & 101.47 & 8.61 & 27.69 & 45.52 & 83.55 & 92.68 & 49.65 \\
\hline S.Em. \pm & 0.2 & 0.14 & 0.06 & 0.05 & 0.05 & 0.06 & 0.01 & 0.08 & 0.05 & 0.04 & 0.15 & 0.13 \\
\hline \multirow[t]{2}{*}{ C.D. at $5 \%$} & NS & 0.40 & 0.18 & 0.16 & 0.15 & 0.18 & 0.04 & 0.25 & 0.17 & 0.14 & 0.45 & 0.40 \\
\hline & \multicolumn{6}{|c|}{ Leaf area plant ${ }^{-1}\left(\mathrm{dm}^{2}\right)$} & \multicolumn{6}{|c|}{ Number of branches plant ${ }^{-1}$} \\
\hline$M_{1}:$ No mulch & 3.65 & 4.88 & 7.22 & 7.72 & 8.16 & 4.15 & 2.25 & 3.18 & 3.47 & 4.86 & 5.48 & 5.48 \\
\hline$M_{2}$ : Soybean straw & 4.40 & 5.41 & 7.56 & 8.23 & 8.53 & 4.37 & 2.37 & 3.19 & 3.68 & 5.12 & 6.72 & 6.72 \\
\hline$M_{3}:$ Sugarcane trash & 4.50 & 5.60 & 7.68 & 8.61 & 8.83 & 4.56 & 2.52 & 3.35 & 3.71 & 5.52 & 6.78 & 6.78 \\
\hline S.Em. \pm & 0.03 & 0.03 & 0.01 & 0.01 & 0.03 & 0.04 & 0.01 & 0.01 & 0.01 & 0.04 & 0.02 & 0.02 \\
\hline \multirow[t]{2}{*}{ C.D. at 5\% } & 0.10 & 0.10 & 0.05 & 0.05 & 0.10 & 0.12 & 0.03 & 0.04 & 0.03 & 0.14 & 0.06 & 0.06 \\
\hline & \multicolumn{6}{|c|}{ Plant spread } & \multicolumn{6}{|c|}{ Dry matter plant $^{-1}(\mathrm{~g})$} \\
\hline$M_{1}:$ No mulch & 14.25 & 20.55 & 29.34 & 38.26 & 40.83 & 41.25 & 1.53 & 5.68 & 11.81 & 17.55 & 19.59 & 19.75 \\
\hline $\mathrm{M}_{2}$ : Soybean straw & 14.69 & 20.95 & 30.23 & 40.73 & 41.97 & 42.54 & 1.86 & 5.92 & 11.88 & 18.04 & 20.06 & 20.21 \\
\hline$M_{3}$ : Sugarcane trash & 14.88 & 21.46 & 31.23 & 41.57 & 43.19 & 44.35 & 2.09 & 5.98 & 12.20 & 18.23 & 20.14 & 20.35 \\
\hline S.Em. \pm & 0.01 & 0.10 & 0.04 & 0.02 & 0.29 & 0.2 & 0.05 & 0.09 & 0.01 & 0.02 & 0.01 & 0.01 \\
\hline \multirow[t]{2}{*}{ C.D. at $5 \%$} & 0.04 & 0.31 & 0.14 & 0.07 & NS & 0.08 & NS & 0.28 & 0.05 & 0.07 & 0.03 & 0.03 \\
\hline & \multicolumn{6}{|c|}{ Number of capsules plant ${ }^{-1}$} & & & & & & \\
\hline$M_{1}$ : No mulch & -- & -- & 25.72 & 40.19 & 55.40 & 55.85 & & & & & & \\
\hline$M_{2}$ : Soybean straw & -- & -- & 27.35 & 41.67 & 56.15 & 56.45 & & & & & & \\
\hline$M_{3}$ : Sugarcane trash & -- & -- & 28.13 & 43.32 & 58.56 & 58.81 & & & & & & \\
\hline S.Em \pm & -- & -- & 0.02 & 0.02 & 0.05 & 0.05 & & & & & & \\
\hline C.D. at $5 \%$ & -- & -- & 0.08 & 0.07 & 0.15 & 0.15 & & & & & & \\
\hline
\end{tabular}

Table.2 Effect of mulches on yield contributing characters and yield of summer sesamum

\begin{tabular}{|c|c|c|c|c|c|c|}
\hline \multirow[b]{2}{*}{ Treatments } & \multirow{2}{*}{$\begin{array}{l}\text { Seed weight } \\
\text { plant }^{-1}(\mathrm{~g})\end{array}$} & \multirow{2}{*}{$\begin{array}{l}\text { Weight of } \\
\text { straw } \\
\text { plant }^{-1}(g)\end{array}$} & \multirow{2}{*}{$\begin{array}{c}\text { Test } \\
\text { Weight } \\
\text { (g) }\end{array}$} & \multicolumn{2}{|c|}{ Yield $\left(\mathrm{kg} \mathrm{ha}^{-1}\right)$} & \multirow{2}{*}{$\begin{array}{c}\text { Harvest } \\
\text { Index }(\%)\end{array}$} \\
\hline & & & & Grain & Straw & \\
\hline$M_{1}:$ No mulch & 3.46 & 11.96 & 3.23 & 764.05 & 1419.45 & 34.97 \\
\hline $\mathrm{M}_{2}$ : Soybean straw & 3.56 & 12.20 & 3.26 & 785.33 & 1439.57 & 35.29 \\
\hline$M_{3}$ : Sugarcane trash & 4.02 & 12.84 & 3.52 & 886.76 & 1574.25 & 36.00 \\
\hline S.Em \pm & 0.05 & 0.04 & 0.02 & 8.9 & 18.70 & - \\
\hline C.D. at 5\% & 0.14 & 0.12 & NS & 25.3 & 56.10 & - \\
\hline
\end{tabular}


Table.3 Economics of sesamum as influenced by different mulches

\begin{tabular}{|c|c|c|c|c|}
\hline Treatment & $\begin{array}{c}\text { Gross } \\
\text { monetary } \\
\text { returns (Rs. } \\
\left.\text { ha }^{-1}\right)\end{array}$ & $\begin{array}{c}\text { Cost of } \\
\text { cultivation } \\
\left(\text { Rs. ha }{ }^{-1}\right)\end{array}$ & $\begin{array}{c}\text { Net } \\
\text { monetary } \\
\text { returns } \\
\left(\text { Rs. ha }{ }^{-1}\right)\end{array}$ & $\begin{array}{l}\text { B:C } \\
\text { ratio }\end{array}$ \\
\hline \multicolumn{5}{|c|}{ B. Sub plot treatments :Mulches } \\
\hline$M_{1}$ : No mulch & 61124 & 43980 & 17144 & 1.38 \\
\hline$M_{2}:$ Soybean straw & 62826 & 36660 & 26166 & 1.71 \\
\hline$M_{3}:$ Sugarcane trash & 70939 & 37160 & 33779 & 1.90 \\
\hline S.Em \pm & 30.45 & - & 31.18 & - \\
\hline C.D. at $5 \%$ & 91.31 & - & 93.47 & - \\
\hline
\end{tabular}

Table.4 Correlation coefficient between weather parameters and plant growth characters

\section{Plant height (cm)}

\begin{tabular}{|c|c|c|c|c|c|c|}
\hline Treatment & $\mathbf{T}_{\max }$ & $\mathbf{T}_{\min }$ & R H-I & R H-II & BSSH & $\begin{array}{l}\text { Canopy } \\
\text { temp. }\end{array}$ \\
\hline $\mathbf{P}_{1} \mathbf{M}_{1}$ & 0.218 & $0.961 * *$ & -0.226 & $0.734 *$ & $-0.737 *$ & $0.976 * *$ \\
\hline$P_{1} M_{2}$ & 0.218 & $0.961^{* *}$ & -0.227 & $0.749 * *$ & $-0.736^{*}$ & $0.976 * *$ \\
\hline$P_{1} M_{3}$ & 0.219 & $0.962 * *$ & -0.281 & $0.749 * *$ & $-0.735^{*}$ & $0.976 * *$ \\
\hline $\mathbf{P}_{2} \mathbf{M}_{1}$ & 0.212 & $0.976^{* *}$ & -0.281 & $0.693 *$ & $-0.689 *$ & $0.986 * *$ \\
\hline $\mathbf{P}_{2} \mathbf{M}_{2}$ & 0.239 & $0.976^{* *}$ & -0.281 & $0.693 *$ & $-0.690 *$ & $0.986 * *$ \\
\hline $\mathbf{P}_{2} \mathbf{M}_{3}$ & 0.279 & 0.980 ** & -0.281 & $0.693 *$ & $-0.690 *$ & $0.986 * *$ \\
\hline $\mathbf{P}_{3} \mathbf{M}_{1}$ & 0.312 & $0.965 * *$ & -0.291 & $0.678^{*}$ & $-0.672 *$ & $0.988^{* *}$ \\
\hline $\mathbf{P}_{3} \mathbf{M}_{2}$ & 0.312 & 0.980 ** & -0.290 & $0.675^{*}$ & $-0.673 *$ & $0.988 * *$ \\
\hline$P_{3} M_{3}$ & 0.311 & $0.980^{* *}$ & -0.290 & $0.679 *$ & $-0.673 *$ & $0.988 * *$ \\
\hline $\mathbf{P}_{4} \mathbf{M}_{1}$ & 0.218 & $0.961 * *$ & -0.226 & $0.728 *$ & $-0.735^{*}$ & $0.976 * *$ \\
\hline $\mathbf{P}_{4} \mathbf{M}_{2}$ & 0.216 & $0.959 * *$ & -0.232 & $0.739 *$ & $-0.738^{*}$ & $0.974 * *$ \\
\hline $\mathbf{P}_{4} \mathbf{M}_{3}$ & 0.218 & $0.961 * *$ & -0.226 & $0.749 * *$ & $-0.735^{*}$ & $0.976 * *$ \\
\hline \multicolumn{7}{|c|}{ Number of functional leaves plant ${ }^{-1}$} \\
\hline $\mathbf{P}_{1} \mathbf{M}_{1}$ & -0.041 & $0.897 * *$ & -0.096 & $\overline{0.674 *}$ & -0.553 & $0.895 * *$ \\
\hline $\mathbf{P}_{1} \mathbf{M}_{2}$ & -0.042 & $0.897^{* *}$ & -0.096 & $0.674 *$ & -0.553 & $0.895 * *$ \\
\hline $\mathbf{P}_{1} \mathbf{M}_{3}$ & -0.035 & $0.898 * *$ & -0.098 & $0.673 *$ & -0.553 & $0.895 * *$ \\
\hline $\mathbf{P}_{2} \mathbf{M}_{1}$ & -0.036 & $0.897 * *$ & -0.107 & $0.667 *$ & -0.546 & $0.895 * *$ \\
\hline $\mathbf{P}_{2} \mathbf{M}_{2}$ & -0.034 & $0.897 * *$ & -0.108 & $0.667 *$ & -0.546 & $0.895 * *$ \\
\hline $\mathbf{P}_{2} \mathbf{M}_{3}$ & -0.035 & $0.896^{* *}$ & -0.105 & $0.667 *$ & -0.546 & $0.894 * *$ \\
\hline $\mathbf{P}_{3} \mathbf{M}_{1}$ & -0.031 & $0.893 * *$ & -0.106 & $0.664 *$ & -0.541 & $0.890 * *$ \\
\hline $\mathbf{P}_{3} \mathbf{M}_{2}$ & -0.035 & $0.892 * *$ & -0.104 & $0.664 *$ & -0.542 & $0.890 * *$ \\
\hline $\mathbf{P}_{3} \mathbf{M}_{3}$ & -0.039 & $0.893 * *$ & -0.105 & $0.664 *$ & -0.541 & $0.890 * *$ \\
\hline $\mathbf{P}_{4} \mathbf{M}_{1}$ & -0.035 & $0.898^{* *}$ & -0.098 & $0.673^{*}$ & -0.553 & $0.895 * *$ \\
\hline $\mathbf{P}_{4} \mathbf{M}_{2}$ & -0.036 & $0.898 * *$ & -0.098 & $0.673^{*}$ & -0.553 & $0.895 * *$ \\
\hline $\mathbf{P}_{4} \mathbf{M}_{3}$ & -0.031 & $0.898 * *$ & -0.099 & $0.673 *$ & -0.552 & $0.896 * *$ \\
\hline \multicolumn{7}{|c|}{ * Significant at $5 \%$} \\
\hline
\end{tabular}




\begin{tabular}{|c|c|c|c|c|c|c|}
\hline \multicolumn{7}{|c|}{ Leaf area plant ${ }^{-1}\left(\mathrm{dm}^{2}\right)$} \\
\hline Treatment & $\mathbf{T}_{\max }$ & $\mathbf{T}_{\min }$ & R H-I & R H-II & BSSH & $\begin{array}{l}\text { Canopy } \\
\text { temp. }\end{array}$ \\
\hline $\mathbf{P}_{1} \mathbf{M}_{1}$ & 0.366 & $0.797 * *$ & -0.320 & 0.247 & -0.096 & $0.752 * *$ \\
\hline $\mathbf{P}_{1} \mathbf{M}_{2}$ & 0.367 & $0.799 * *$ & -0.322 & 0.247 & -0.097 & $0.754 * *$ \\
\hline $\mathbf{P}_{1} \mathbf{M}_{3}$ & 0.370 & $0.799 * *$ & -0.323 & 0.245 & 0.095 & $0.754 * *$ \\
\hline $\mathbf{P}_{2} \mathbf{M}_{1}$ & 0.368 & $0.743^{*}$ & -0.279 & 0.209 & -0.043 & $0.693^{*}$ \\
\hline $\mathbf{P}_{2} \mathbf{M}_{2}$ & 0.372 & $0.745^{*}$ & -0.285 & 0.207 & -0.042 & $0.695^{*}$ \\
\hline $\mathbf{P}_{2} \mathbf{M}_{3}$ & 0.373 & $0.745^{*}$ & -0.285 & 0.208 & -0.041 & $0.695^{*}$ \\
\hline $\mathbf{P}_{3} \mathbf{M}_{1}$ & 0.345 & $0.755 * *$ & -0.263 & 0.242 & -0.070 & $0.707^{*}$ \\
\hline $\mathbf{P}_{3} \mathbf{M}_{2}$ & 0.352 & $0.753 * *$ & -0.269 & 0.235 & -0.063 & $0.705^{*}$ \\
\hline $\mathbf{P}_{\mathbf{3}} \mathbf{M}_{3}$ & 0.347 & $0.756 * *$ & -0.266 & 0.241 & -0.069 & $0.708^{*}$ \\
\hline $\mathbf{P}_{4} \mathbf{M}_{1}$ & 0.364 & $0.798 * *$ & -0.318 & 0.250 & -0.099 & $0.754 * *$ \\
\hline $\mathbf{P}_{4} \mathbf{M}_{2}$ & 0.359 & $0.801 * *$ & -0.315 & 0.255 & -0.105 & $0.756^{* *}$ \\
\hline $\mathbf{P}_{4} \mathbf{M}_{3}$ & 0.366 & $0.797 * *$ & -0.320 & 0.247 & -0.096 & $0.752 * *$ \\
\hline \multicolumn{7}{|c|}{ Number of branches plant ${ }^{-1}$} \\
\hline $\mathbf{P}_{1} \mathbf{M}_{1}$ & -0.142 & $0.890^{* *}$ & -0.192 & $0.762 * *$ & $-0.719^{*}$ & $0.863 * *$ \\
\hline $\mathbf{P}_{1} \mathbf{M}_{2}$ & -0.145 & $0.891 * *$ & -0.192 & $0.762^{* *}$ & $-0.719 *$ & $0.864 * *$ \\
\hline $\mathbf{P}_{\mathbf{1}} \mathbf{M}_{\mathbf{3}}$ & -0.144 & $0.892 * *$ & -0.196 & $0.760 * *$ & $-0.717^{*}$ & $0.865^{* *}$ \\
\hline $\mathbf{P}_{2} \mathbf{M}_{1}$ & -0.316 & $0.817 * *$ & -0.074 & $0.843^{* *}$ & $-0.830 * *$ & $0.783^{* *}$ \\
\hline $\mathbf{P}_{2} \mathbf{M}_{2}$ & -0.319 & $0.818 * *$ & -0.078 & $0.841 * *$ & $-0.827 * *$ & $0.784 * *$ \\
\hline $\mathbf{P}_{2} \mathbf{M}_{3}$ & -0.318 & $0.818 * *$ & -0.077 & $0.842 * *$ & $-0.826 * *$ & $0.784 * *$ \\
\hline $\mathbf{P}_{3} \mathbf{M}_{1}$ & -0.315 & $0.818 * *$ & -0.076 & $0.841^{* *}$ & $-0.821 * *$ & $0.785^{* *}$ \\
\hline $\mathbf{P}_{3} \mathbf{M}_{2}$ & -0.355 & $0.821 * *$ & -0.082 & $0.838^{* *}$ & $-0.817 * *$ & $0.788 * *$ \\
\hline $\mathbf{P}_{\mathbf{3}} \mathbf{M}_{3}$ & -0.32 & $0.821 * *$ & -0.082 & $0.839 * *$ & $-0.819 * *$ & $0.788 * *$ \\
\hline $\mathbf{P}_{4} \mathbf{M}_{1}$ & -0.144 & $0.889 * *$ & -0.192 & $0.762^{* *}$ & $-0.720 *$ & $0.862 * *$ \\
\hline $\mathbf{P}_{4} \mathbf{M}_{2}$ & -0.132 & $0.893 * *$ & -0.200 & $0.757 * *$ & $-0.714^{*}$ & $0.866^{* *}$ \\
\hline $\mathbf{P}_{4} \mathbf{M}_{3}$ & -0.136 & $0.892 * *$ & -0.199 & $0.757^{* *}$ & $-0.714^{*}$ & $0.865 * *$ \\
\hline \multicolumn{7}{|c|}{ Plant spread $(\mathrm{cm})$ plant $^{-1}$} \\
\hline
\end{tabular}

\begin{tabular}{|c|c|c|c|c|c|c|}
\hline Treatment & Tmax. & Tmin. & RH-I & RH-II & BSS & $\begin{array}{c}\text { Canopy } \\
\text { Temp. }\end{array}$ \\
\hline $\mathbf{P}_{\mathbf{1}} \mathbf{M}_{\mathbf{1}}$ & -0.189 & $0.925^{* *}$ & -0.106 & $0.808^{* *}$ & $-0.780^{* *}$ & $0.946^{* *}$ \\
\hline $\mathbf{P}_{\mathbf{1}} \mathbf{M}_{\mathbf{2}}$ & -0.186 & $0.925^{* *}$ & -0.107 & $0.807^{* *}$ & $-0.789^{* *}$ & $0.946^{* *}$ \\
\hline $\mathbf{P}_{\mathbf{1}} \mathbf{M}_{\mathbf{3}}$ & -0.173 & $0.926^{* *}$ & -0.107 & $0.807^{* *}$ & $-0.779^{* *}$ & $0.946^{* *}$ \\
\hline $\mathbf{P}_{\mathbf{2}} \mathbf{M}_{\mathbf{1}}$ & -0.168 & $0.928^{* *}$ & -0.100 & $0.804^{* *}$ & $-0.768^{* *}$ & $0.947^{* *}$ \\
\hline $\mathbf{P}_{\mathbf{2}} \mathbf{M}_{\mathbf{2}}$ & -0.165 & $0.928^{* *}$ & -0.100 & $0.804^{* *}$ & $-0.769^{* *}$ & $0.947^{* *}$ \\
\hline $\mathbf{P}_{\mathbf{2}} \mathbf{M}_{\mathbf{3}}$ & -0.162 & $0.929^{* *}$ & -0.100 & $0.804^{* *}$ & $-0.768^{* *}$ & $0.948^{* *}$ \\
\hline $\mathbf{P}_{\mathbf{3}} \mathbf{M}_{\mathbf{1}}$ & -0.187 & $0.921^{* *}$ & -0.095 & $0.810^{* *}$ & $-0.779^{* *}$ & $0.942^{* *}$ \\
\hline $\mathbf{P}_{\mathbf{3}} \mathbf{M}_{\mathbf{2}}$ & -0.184 & $0.920^{* *}$ & -0.095 & $0.811^{* *}$ & $-0.780^{* *}$ & $0.942^{* *}$ \\
\hline $\mathbf{P}_{\mathbf{3}} \mathbf{M}_{\mathbf{3}}$ & -0.181 & $0.920^{* *}$ & -0.096 & $0.812^{* *}$ & $-0.781^{* *}$ & $0.941^{* *}$ \\
\hline $\mathbf{P}_{\mathbf{4}} \mathbf{M}_{\mathbf{1}}$ & -0.180 & $0.925^{* *}$ & -0.106 & $0.808^{* *}$ & $-0.780^{* *}$ & $0.946^{* *}$ \\
\hline $\mathbf{P}_{\mathbf{4}} \mathbf{M}_{\mathbf{2}}$ & -0.182 & $0.925^{* *}$ & -0.106 & $0.807 * *$ & $-0.779^{* *}$ & $0.946^{* *}$ \\
\hline $\mathbf{P}_{\mathbf{4}} \mathbf{M}_{\mathbf{3}}$ & -0.184 & $0.925^{* *}$ & -0.106 & $0.807^{* *}$ & $-0.779^{* *}$ & $0.946^{* *}$ \\
\hline
\end{tabular}

** Significant at $1 \% *$ Significant at $5 \%$ 


\begin{tabular}{|c|c|c|c|c|c|c|}
\hline \multicolumn{7}{|c|}{ Table.6 Correlation coefficient between weather parameters and plant growth characters } \\
\hline \multicolumn{7}{|c|}{ Number of capsules plant ${ }^{-1}$} \\
\hline Treatment & $\mathbf{T}_{\max }$ & $\mathbf{T}_{\min }$ & R H-I & R H-II & BSSH & Canopy temp. \\
\hline $\mathbf{P}_{1} \mathbf{M}_{1}$ & $0.654 *$ & $0.977 * *$ & -0.454 & 0.412 & -0.420 & $0.966 * *$ \\
\hline $\mathbf{P}_{1} \mathbf{M}_{2}$ & $0.655^{*}$ & $0.977 * *$ & -0.455 & 0.410 & -0.418 & $0.966^{* *}$ \\
\hline$P_{1} M_{3}$ & $0.656^{*}$ & $0.977^{* *}$ & -0.456 & 0.409 & -0.417 & $0.966^{* * *}$ \\
\hline $\mathbf{P}_{2} \mathbf{M}_{1}$ & $0.659^{*}$ & $0.976^{* *}$ & -0.459 & 0.401 & -0.411 & $0.965^{* *}$ \\
\hline $\mathbf{P}_{2} \mathbf{M}_{2}$ & $0.658^{*}$ & $0.976^{* *}$ & -0.458 & 0.403 & -0.413 & $0.965^{* * *}$ \\
\hline $\mathbf{P}_{2} \mathbf{M}_{3}$ & $0.659^{*}$ & $0.976^{* *}$ & -0.459 & 0.402 & -0.412 & $0.965^{* * *}$ \\
\hline $\mathbf{P}_{3} \mathbf{M}_{1}$ & $0.671^{*}$ & $0.979 * *$ & -0.474 & 0.392 & -0.399 & $0.969 * *$ \\
\hline $\mathbf{P}_{3} \mathbf{M}_{2}$ & $0.671^{*}$ & 0.980 ** & -0.475 & 0.390 & -0.399 & $0.969 * *$ \\
\hline $\mathbf{P}_{3} \mathbf{M}_{3}$ & $0.672 *$ & $0.980 * *$ & -0.475 & 0.393 & -0.399 & $0.970 * *$ \\
\hline $\mathbf{P}_{4} \mathbf{M}_{1}$ & $0.655^{*}$ & $0.977 * *$ & -0.455 & 0.410 & -0.418 & $0.966^{* *}$ \\
\hline $\mathbf{P}_{4} \mathbf{M}_{2}$ & $0.655^{*}$ & $0.977 * *$ & -0.455 & 0.410 & -0.418 & $0.966 * *$ \\
\hline $\mathbf{P}_{4} \mathbf{M}_{3}$ & $0.655^{*}$ & $0.977^{* *}$ & -0.456 & 0.409 & -0.417 & $0.966 * *$ \\
\hline \multicolumn{7}{|c|}{ Dry matter plant ${ }^{-1}(\mathrm{~g})$} \\
\hline $\mathbf{P}_{1} \mathbf{M}_{1}$ & -0.228 & $0.930^{* *}$ & -0.100 & $0.814^{* *}$ & $-0.784 * *$ & $0.922 * *$ \\
\hline$P_{1} M_{2}$ & -0.225 & $0.930^{* *}$ & -0.100 & $0.814^{* *}$ & $-0.785 * *$ & $0.921 * *$ \\
\hline $\mathbf{P}_{1} \mathbf{M}_{3}$ & -0.222 & 0.930 ** & -0.101 & $0.814 * *$ & $-0.784 * *$ & $0.922 * *$ \\
\hline $\mathbf{P}_{2} \mathbf{M}_{1}$ & -0.224 & $0.928 * *$ & -0.097 & $0.816^{* *}$ & $-0.784 * *$ & $0.920 * *$ \\
\hline $\mathbf{P}_{2} \mathbf{M}_{2}$ & -0.227 & $0.928 * *$ & -0.096 & $0.816^{* *}$ & $-0.785 * *$ & $0.920 * *$ \\
\hline $\mathbf{P}_{2} \mathbf{M}_{3}$ & -0.221 & $0.929 * *$ & -0.097 & $0.816^{* *}$ & $-0.784 * *$ & $0.921 * *$ \\
\hline $\mathbf{P}_{3} \mathbf{M}_{1}$ & -0.210 & $0.931 * *$ & -0.101 & $0.812 * *$ & $-0.782 * *$ & $0.922 * *$ \\
\hline $\mathbf{P}_{3} \mathbf{M}_{2}$ & -0.212 & $0.931 * *$ & -0.100 & $0.813^{* *}$ & $-0.782 * *$ & $0.922 * *$ \\
\hline $\mathbf{P}_{3} \mathbf{M}_{3}$ & -0.213 & $0.930^{* *}$ & -0.099 & $0.813^{* *}$ & $-0.782 * *$ & $0.922 * *$ \\
\hline $\mathbf{P}_{4} \mathbf{M}_{1}$ & -0.226 & $0.93 * *$ & -0.101 & $0.814 * *$ & $-0.783 * *$ & $0.922 * *$ \\
\hline $\mathbf{P}_{4} \mathbf{M}_{2}$ & -0.225 & $0.930^{* *}$ & -0.101 & $0.813 * *$ & $-0.784 * *$ & $0.922 * *$ \\
\hline $\mathbf{P}_{4} \mathbf{M}_{3}$ & -0.221 & $0.930 * *$ & -0.101 & $0.814 * *$ & $-0.783 * *$ & $0.922 * *$ \\
\hline \multicolumn{7}{|c|}{ * Significant at $5 \%$} \\
\hline
\end{tabular}

\section{Economics study}

\section{Gross monetary returns (Rs. ha' $\left.{ }^{-1}\right)$}

Gross monetary returns were significantly affected by different mulch (Table 3). treatments. Maximum gross monetary returns (Rs $70939 \mathrm{ha}^{-1}$ ) were obtained with the application of sugarcane trash mulch @ $5 \mathrm{t} \mathrm{ha}^{-}$ ${ }^{1}$ which was superior over rest of the mulch treatments.

\section{Net monetary returns (Rs. ha $\left.{ }^{-1}\right)$}

Net monetary returns were significantly affected by different mulch treatments (Table 3). Maximum net monetary returns (Rs. 33779 $\mathrm{ha}^{-1}$ ) were obtained when the mulching was done with sugarcane trash mulch @ $5 \mathrm{t} \mathrm{ha}^{-1}$ and it was found significantly superior over the rest of the mulch treatments. Similar findings were reported by Shinde and Firake (1998).

\section{Cost of cultivation}

Under different mulch treatments the cost of cultivation (Rs. $43980 \mathrm{ha}^{-1}$ ) was higher with the no mulch treatment as compared to rest of the treatment (Table 3).

\section{Benefit: Cost ratio}

Under different mulch treatments, the benefit: cost ratio was higher with the application of 
sugarcane trash mulch @ $5 \mathrm{t} \mathrm{ha}^{-1}$ i.e. (1.90) as compared with the soybean straw mulch (1.71) and no mulch (1.38), (Table 3) Similar results were reported by Sutagundi (2000) and Shinde et al., (1998). The maximum gross and net monetary returns under ridges and furrows at $45 \times 10 \mathrm{~cm}^{2}$ with sugarcane trash mulch @ $5 \mathrm{t} \mathrm{ha}^{-1}$ finally showed the higher B : C ratio.

\section{Correlation study}

Correlation coefficient between weather parameters and plant growth contributing characters of summer sesamum

\section{Plant height (cm)}

Maximum temperature has been positively correlated with plant height in respect of all the treatments. However, it is indicating that increase in maximum temperature increased plant height (Table 4). Minimum temperature has been positively correlated with plant height in respect of all the treatments. However, it was indicated that increase in minimum temperature increased plant height and vice-versa. RH-I was found significantly negative correlated with plant height in respect of all the treatments. However, it was indicated that decrease in $\mathrm{RH}-\mathrm{I}$ increased plant height.

RH-II was significantly positive correlated with plant height in respect of all the treatments. However, it was indicated that increase in RH-II increased plant height and vice-versa (Table 4). Bright sunshine hours have been negatively correlated with plant height in respect of all the treatments. However, it is indicating that decrease in Bright sunshine hours increased plant height and vice-versa (Table 4). Canopy temperature has been positively correlated with plant height in respect of all the treatments. However, it was indicated that increase in canopy temperature increased plant height or vice-versa

\section{Number of functional leaves plant ${ }^{-1}$}

Minimum temperature, RH-I, bright sunshine hours and canopy temperature were positively correlated with number of functional leaves in respect of all the treatments. However, it is indicating that increase in minimum temperature, RH-II, and canopy temperature increased number of functional leaves and vice-versa except maximum temperature, $\mathrm{RH}$ I, and BSS were found negatively correlated with number of leaves in all the treatments (Table 4).

\section{Leaf area plant ${ }^{-1}$}

Both maximum and minimum temperature, RH- II and canopy temperature were positively correlated with leaf area in respect of all the treatments. However, it was indicated that increase in maximum temperature, minimum temperature, $\mathrm{RH}-\mathrm{II}$ and canopy temperature increased the leaf area and vice-versa. Except RH-I and bright sunshine hours was negatively correlated with number of leaf area in respect of all the treatments (Table 5).

\section{Number of branches plant ${ }^{-1}$}

Minimum temperature, RH-II and canopy temperature were found positively correlated with number of branches in respect of all the treatments. However, it was indicated that increase in minimum temperature, RH-I, and canopy temperature increased number of branches or vice-versa (Table 5).

Maximum temperature, RH-I and bright sunshine hours were found negatively correlated with number of branches in respect of all the treatments. However, it was indicated that decrease maximum temperature and bright sunshine hours increased number of branches or vice-versa (Table 5). 


\section{Plant spread (cm) plant ${ }^{-1}$}

Minimum temperature, RH-II, bright sunshine hours and canopy temperature were positively correlated with plant spread in respect of all the treatments. However, it was indicated that increase of minimum temperature, $\mathrm{RH}-\mathrm{II}$ and canopy temperature increased plant spread and vice-versa (Table 5).

\section{Number of capsules plant ${ }^{-1}$}

Maximum temperature, minimum temperature, RH-II and canopy temperature were positively correlated with number of capsules in respect of all the treatments. However, it was indicating that increase maximum temperature, minimum temperature, RH-II and canopy temperature increased number of capsules and vice-versa (Table 6).

RH I and bright sunshine hours was negatively correlated with number of capsules in respect of all the treatments. However, it was indicated that decrease RH-I and bright sunshine hours increased number of capsules or vice-versa (Table 6).

\section{Dry matter plant ${ }^{-1}(g)$}

Minimum temperature, RH-II, bright sunshine hours and canopy temperature were positively correlated with dry matter production in respect of all the treatments. However, it was indicated that increase in minimum temperature, RH-II and canopy temperature increased dry matter and vice-versa (Table 6). Maximum temperature and RH-I was negatively correlated with dry matter production in respect of all the treatments.

However, it is indicating that decrease of maximum temperature and bright sunshine hours increased dry matter or vice-versa (Table 6).

\section{References}

Anisuzzaman, M., Ashrafuzzaman, M., Ismail, M.R., Uddin, M.K. and Rahim M.A. 2009. Planting time and mulching effect on onion development and seed production. African J. Biotech. 8(3): 412-416.

Anonymous, 1997. Annual Research Report on sesamum and linseed. Agronomy and Soil Science, 1997-98. pp. 19-21.

Anonymous, 2012.Agriculture statistics at glance (ICAR publication).pp 83-85.

Ghosh, P.K., Devidayal, S.V., Naik, P. R., Dayal, D. and Singh, V. 1997 Improvement of yield of summer groundnut through mulching and criss cross sowing in Gujarat, India. International Arachis News-letter: 6162.

Katmale, S.N. 2000.yield maximization in summer groundnut by using polythele mulch J. Karnataka Aric. Univ., 9(1):12-19.

Kumar, S. and Goel, S.C. 1994.Population dynamics of a pyralid, Antigastra catalaunalis (Dup.) on sesamum in relation to abiotic factors. J. Ento. Res. 18 (1): 61-64.

Pawar, S.N., Kalbande, S.R. and Jadhav S.B. 2008. Effect of moisture conservation techniques on growth and yield of summer groundnut. Intern. J. Agric.Sci. 4(1): 119-123.

Shinde, U.R. and Firake, N. N. 1998. Economics of summer chilli production with mulching and micro irrigation. J Mahhashtra Agric. Univ., 23(1): 14-16.

Sonawane, V.P. 2007. Studies on physiochemical and microbiological properties of soil as influenced by different mulches on rabi onion cv. N2-4-1.M.Sc. (Agri) thesis submitted to M.P.K.V., Rahuri (Maharashtra). 
Sutagundi, R. H., 2000. Effect of mulches and manures on growth and yield of chilli (Capsicum annum L.). M. Sc. (Agri.) Thesis, Univ. Agric. Sci. Dharwad, Karnataka (India).
Yadav, S.T. and Patil, P.P. 2009. Response of summer capsicum yield to different mulching in western Ghat zone of Maharashtra. J. Agrometeorology. 11(1): 83-84.

\section{How to cite this article:}

Mundhe, S. G., D. A. Sonawane and Suryavanshi, P. B. 2019. The Significance of Mulches on Growth, Yield and Quality of Summer Sesamum (Sesamum indicum L.) in Relation to Weather Parameters. Int.J.Curr.Microbiol.App.Sci. 8(10): 462-472.

doi: https://doi.org/10.20546/ijcmas.2019.810.050 